\title{
Diseño de una red de monitorización de variables meteorológicas relacionadas a los tornados en Barranquilla-Colombia y su área metropolitana
}

\author{
Design of a monitoring network of meteorological variables related to \\ tornadoes in Barranquilla-Colombia and its metropolitan area
}

\author{
Alejandro Cama-Pinto ${ }^{1} \quad$ Gabriel Piñeres-Espitia ${ }^{1} \quad$ Zhoe Comas-González $^{1 *}$ \\ Jaime Vélez-Zapata ${ }^{1} \quad$ Francisco Gómez-Mula ${ }^{2}$ \\ Recibido 4 de diciembre de 2015, aceptado 3 de octubre de 2016 \\ Received: December 4, 2015 Accepted: October 3, 2016
}

\begin{abstract}
RESUMEN
El presente trabajo muestra la incidencia de los tornados más representativos registrados desde el 1993 en el departamento del Atlántico ubicado al norte de Colombia que ha dejado grandes pérdidas materiales y económicas. Ante ello, se propone el diseño de un prototipo de sistema de monitorización de variables meteorológicas asociadas a la aparición de tornados en zonas donde se han presentado estos fenómenos atmosféricos para que los valores almacenados sirvan para su estudio y posterior predicción. Se emplea una red de sensores inalámbricos compuesta por cuatro nodos y un sumidero usando la plataforma Waspmote y módulos Xbee-PRO ZB (S2) para adquisición de datos y transmisión de la información. Se muestra la ubicación los nodos y la estabilidad de los datos obtenidos por los diferentes sensores del sistema.
\end{abstract}

Palabras clave: Redes de sensores inalámbricas, sistemas de alertas tempranas, tornados en Barranquilla, monitorización atmosférica, sistemas de telecomunicaciones.

\begin{abstract}
This paper shows the incidence of the most representative tornadoes since 1993 registered on the Atlantico's department, located in the north of Colombia; they have left thousands of material and economic losses. For this reason, it is proposed the design of a prototype system for monitoring meteorological variables associated with the occurrence of tornadoes in areas where they have occurred, to use the registered values in its study and following prediction. This system is based in a wireless sensor network, comprised of four sensor nodes and a sink node using the Waspmote platform and Xbee-PRO ZB (S2) modules for data acquisition and transmission respectively. The location of nodes and the stability of the data obtained by the different sensors in the system are also showed in the current work.
\end{abstract}

Keywords: Wireless sensor network (WSN), Early Warning System (EWS), tornadoes in Barranquilla, atmosphere monitoring, Telecommunication systems.

1 Facultad de Ingeniería. Universidad de la Costa. Calle 58 \# 55-66. Barranquilla, Colombia. E-mail: acama1 @ cuc.edu.co; gpineres1@cuc.edu.co; zcomas1@cuc.edu.co; jvelez@cuc.edu.co

2 Escuela Técnica Superior de Ingenierías Informática y de Telecomunicación. Universidad de Granada. Calle Periodista Daniel Saucedo Aranda, s/n, 18071. Granada, España. E-mail: frgomez@ugr.es

* Autor de correspondencia. 


\section{INTRODUCCIÓN}

Un tornado comprende una columna de aire que gira violentamente hacia abajo en forma de embudo, tirando polvo y escombros cuando va transfiriendo su energía a lo largo del camino que recorre [1-2]; es uno de los fenómenos más extremos de la naturaleza con alto potencial de daño localizado y puede ocurrir en cualquier momento del año [3] incrementándose a nivel mundial como resultado del cambio climático [4]. Este evento meteorológico ocurre en pocos cientos de metros y tiene un tiempo de vida de pocos minutos a horas pudiendo llegar a ocasionar catástrofes, perjuicios económicos e incluso pérdida de vidas humanas [5-6]. Se han registrado en todos los continentes a excepción de la Antártida, siendo Norteamérica la zona del planeta con el mayor número contabilizado [7].

No obstante, los tornados se producen generalmente entre las latitudes de $20^{\circ}$ y $60^{\circ}$ en ambos hemisferios [8], siendo una excepción Barranquilla por estar fuera de este rango (latitud norte: $11^{\circ} 01^{\prime}$ y longitud oeste: $\left.74^{\circ} 49^{\prime}\right)$. Barranquilla es una de las principales ciudades de Colombia y tiene una población de más de un millón de habitantes [9], con una temperatura promedio anual de $28^{\circ} \mathrm{C}$ y una humedad relativa de $80 \%$ con solo dos tipos de estaciones, la seca, que va de diciembre a marzo y la húmeda el resto del año; en esta última se origina una alta humedad relativa con abundantes lluvias y tormentas [10]. Y se han registrado tornados de niveles F0 hasta F2 en la escala de Fuijta (T1 a T4 en la escala de Torro), algunos de proporciones moderadas [11-13] considerados de tipo mesociclónico. Uno de los más intensos [14] ocurrió en el 2006, siendo catalogado en el nivel EF2 o T4 dada la magnitud de los daños que ocasionó. En la Figura 1 aparecen imágenes de diferentes tornados ocurridos en el 2010.

Ante este fenómeno natural, se propone el uso de un prototipo de red de sensores inalámbricos (Wireless Sensor Networks - WSN) para monitorear variables ambientales asociada a los tornados que permitan analizar las condiciones meteorológicas antes, durante y después de su aparición en las zonas de Barranquilla y su área metropolitana donde han sido registradas.

Se usa la tecnología WSN por su rápida implementación y configuración, además porque

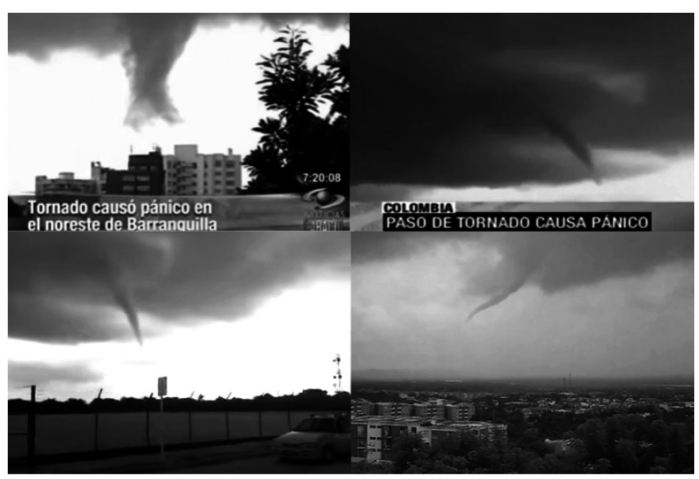

Figura 1. Registro de tornados en la ciudad de Barranquilla.

Fuente: https://youtu.be/Bdm871J560s https://youtu.be/HGz4T5WOg8o.

simplifica la integración de los sensores y el sistema de alimentación que favorece a la estabilidad en la medición de las variables asociadas con las condiciones meteorológicas que se presentan cuando se produce un tornado. Hay trabajos que utilizan la tecnología WSN pero con enfoques distintos, como el expuesto por [15] en donde se muestra un sistema para monitoreo agrícola basado en plataformas de hardware y software abiertas. Así mismo en [16] se plantea un sistema de monitoreo de variables para arroyos que se forman en las calles de Barranquilla, utilizando estaciones similares. En [17] se realizan estudios urbanos para monitoreo de condiciones locales de calidad de aire en Chile, mediante el uso de estaciones ambientales. En [18] se usa en aplicaciones IoT (Internet de las cosas), también para el monitoreo de variables ambientales y meteorológicas locales.

\section{TORNADOS EN BARRANQUILLA}

Estudios realizados por entidades como el IDEAM (Instituto de Hidrología, Meteorología y Estudios Ambientales en Colombia), CACOM3 (Comando Aéreo de Combate $\mathrm{N}^{\circ} 3$ de la Fuerza Aérea Colombiana) y la Universidad de la Costa, han demostrado que la ciudad de Barranquilla y municipios aledaños dentro del departamento del Atlántico como Soledad, Malambo y Sabanalarga, han sido impactados por vendavales, coletazos de huracanes, y fuertes precipitaciones. Estos eventos se recopilan desde el 1993 y se muestran en la Tabla 1 y en 
[19] [20] con los registros de tornados y microtornados en la ciudad de Barranquilla y municipios aledaños con base en la información periodística consignada en diferentes diarios del país así como por medio de fuentes de entidades gubernamentales; entre los daños más comunes se tienen las tejas expelidas de los techos, árboles arrancados de la raíz y líneas eléctricas caídas. Estos fenómenos han sido clasificados de acuerdo a las escalas de Fujita ampliada y Torro [21] en la Tabla 1, actualizada al 2016.

\section{SISTEMAS DE MONITOREO PARA TORNADOS}

Aunque los tornados en el departamento del Atlántico no se pueden comparar con otras regiones del mundo donde son más destructivos, es necesaria la existencia de un sistema de monitoreo que permita generar alertas a la población vulnerable a este tipo de fenómenos, por los riesgos que representan los antecedentes expuestos. Se revisaron diferentes tecnologías empleadas en la actualidad, destacándose los estudios basados en la asociación de movimientos sísmicos provocados por la transferencia de la energía de los tornados cuando tocan tierra [28] o mediante el "sensado social", que actúa como una red de sensores conformada por la información de las personas por medio de las clásicas observaciones meteorológicas [29-30]. Sin embargo, entre las tecnologías más difundidas actualmente están las que emplean el radar Doppler para predecir y detectar la aparición de tornados en su fase inicial. Esto es

Tabla 1. Registro de tornados y sus secuelas en el departamento del Atlántico, Colombia [14] [19].

\begin{tabular}{|c|c|c|c|c|}
\hline \multirow{2}{*}{$\begin{array}{l}\text { Heridos y } \\
\text { damnificados } \\
\text { reportados }\end{array}$} & \multirow{2}{*}{ Daños materiales } & \multicolumn{2}{|c|}{$\begin{array}{l}\text { Escala de } \\
\text { tornado }\end{array}$} & \multirow{2}{*}{ Lugar y fecha } \\
\hline & & Fujita & Torro & \\
\hline No hay registro & $\begin{array}{l}\text { Árboles arrancados de raíz, daños de fluido } \\
\text { eléctrico, edificaciones averiadas }\end{array}$ & EF0 & $\mathrm{T} 2$ & Barranquilla, mayo de 1993. \\
\hline 6.000 damnificados & Viviendas afectadas, techos expulsados & EF1 & $\mathrm{T} 2$ & Soledad, junio del 2001. \\
\hline No hay registro & Amenaza de tornado & NA & NA & Barranquilla, agosto del 2003. \\
\hline 19 heridos & $\begin{array}{l}\text { Casas sin techos, árboles arrancados de raíz, casas } \\
\text { de poca cimentación expelidas, millonarios daños. }\end{array}$ & $\mathrm{EF} 2$ & $\mathrm{~T} 4$ & Barranquilla, septiembre del 2006. \\
\hline 12 heridos & 158 edificaciones destechadas & EF0 & $\mathrm{T} 1$ & Barranquilla, junio del 2007. \\
\hline $\begin{array}{l}18 \text { heridos, } \\
528 \text { damnificados }\end{array}$ & $\begin{array}{l}413 \text { viviendas afectadas, techos expelidos, árboles } \\
\text { arrancados de raíz, muros derrumbados, millonarios } \\
\text { daños. }\end{array}$ & EF0 & T1 & Soledad, mayo del 2008. \\
\hline $\begin{array}{l}\text { No hay registro. } \\
\text { Coletazo de tornado }\end{array}$ & Láminas de techos expelidas & NA & NA & Soledad, julio del 2010. \\
\hline No hay registro & Amenaza de tornado & NA & NA & Soledad, agosto del 2010. \\
\hline 600 damnificados & 116 casas destechadas. Líneas eléctricas caídas & EF1 & $\mathrm{T} 2$ & Barranquilla, septiembre del 2010. \\
\hline No hay registros & Amenaza de tornado & NA & NA & Barranquilla, octubre del 2010. \\
\hline 36 damnificados & $\begin{array}{l}36 \text { viviendas destruidas y múltiples enseres } \\
\text { completamente averiados }\end{array}$ & EF0 & $\mathrm{T} 1$ & Piojó, mayo del 2011. \\
\hline 170 damnificados & $\begin{array}{l}\text { Casas destechadas, paredes caídas, árboles } \\
\text { arrancados }\end{array}$ & EF0 & $\mathrm{T} 2$ & Ponedera, septiembre del 2012 [22]. \\
\hline $\begin{array}{l}1 \text { muerto y } \\
107 \text { damnificados }\end{array}$ & $\begin{array}{l}\text { Casas destechadas, paredes caídas, líneas eléctricas } \\
\text { caídas, árboles arrancados }\end{array}$ & EF1 & $\mathrm{T} 2$ & Sabanalarga, junio del 2012 [23]. \\
\hline 190 damnificados & $\begin{array}{l}\text { Casas destechadas, paredes caídas, árboles } \\
\text { arrancados }\end{array}$ & EF0 & $\mathrm{T} 2$ & Soledad, septiembre del 2013 [24]. \\
\hline $\begin{array}{l}\text { No hay registro. } \\
\text { Coletazo de tornado }\end{array}$ & Amenaza de tornado & NA & NA & Barranquilla, noviembre del 2014 [25]. \\
\hline 92 damnificados & Casas destechadas & EF0 & $\mathrm{T} 1$ & $\begin{array}{l}\text { Sabanalarga - Ponedera, septiembre } \\
\text { del } 2015 \text { [26]. }\end{array}$ \\
\hline $\begin{array}{l}36 \text { damnificados. } \\
1 \text { herido }\end{array}$ & Casas destechadas & EF0 & $\mathrm{T} 1$ & Barranquilla, abril del 2016 [27]. \\
\hline
\end{tabular}


conocido como tornado-génesis, cuyo análisis revela si es inminente minutos antes que se produzca [3132], además sondea el interior de las tormentas y mide los cambios de viento peligrosos; por ello su uso es muy difundido en los aeropuertos [33]. Un inconveniente que presenta este tipo de tecnología es que no siguen la curvatura de la tierra lo que afecta la exactitud de las mediciones [34]. Este tipo de radares meteorológicos es usado en la NOAA (National Oceanic and Atmospheric Administration) debido a que en EE.UU. se presentan tornados con bastante frecuencia en distintas zonas de su territorio. Otro ejemplo es empleando este sistema de forma móvil, montándolos en vehículos y yendo a la persecución de los tornados para recoger datos de su trayectoria y peligrosidad [35].

Para monitorizar las condiciones ambientales y meteorológicas que se suscitan cuando se presentan los tornados, también se usan las imágenes obtenidas por satélites meteorológicos como el Meteosat, cuyo radiómetro opera en la banda espectral de los infrarrojos para analizar las características de las masas de aire atmosféricas [8, 36-38].

Por último, otra tecnología que ha ido ganando terreno en los últimos años y que compete al desarrollo de este artículo son las redes de sensores inalámbricos (WSN), cuyos méritos son el bajo costo de los nodos sensores, la rápida respuesta y capacidad para capturar y reenviar información con mínimo retardo, aparte de su escalabilidad y flexibilidad en el despliegue que la convierte en un sistema idóneo para monitorizar este tipo de eventos naturales que aparecen de forma sucinta [39-41], dado que pueden detectar o predecir variadas condiciones meteorológicas locales que se presentan en determinadas regiones.

\section{DISEÑO DE UNA RED DE MONITOREO PARA TORNADOS}

Para desarrollar el diseño de la red de monitoreo de las condiciones meteorológicas donde se han presentado tornados, se usó la metodología WBS (Work Breakdown Structure) [42]. Y con el fin de estructurar los alcances se proponen cuatro fases derivadas en actividades.

En la primera fase realizamos un análisis a los antecedentes de los tornados en Barranquilla y su área metropolitana, determinando la incidencia de los fenómenos atmosféricos en el departamento del Atlántico y realizando un estudio sobre las condiciones meteorológicas locales.

La segunda fase evalúa los sistemas (equipos y herramientas) más comunes de monitoreo para tornados, sustentado en la revisión bibliográfica.

En la tercera fase se caracteriza y selecciona la plataforma tecnológica del sistema de monitoreo, con base en las condiciones meteorológicas locales que generan tornados.

La cuarta y última fase consiste en la propuesta de una red de sensores inalámbricos como sistema de monitoreo para los tornados.

\section{Evaluación y selección de tecnologías}

Para la medición de las variables atmosféricas como temperatura, cantidad de lluvia, humedad relativa, presión atmosférica, velocidad y dirección del viento, se necesita de una plataforma de adquisición de datos que almacene la información tomada del ambiente. Para dicho fin, se eligió por su versatilidad y practicidad en el despliegue, la implementación de una red de sensores inalámbricos (WSN - Wireless Sensor Networks) en los lugares de mayor índice de prevalencia e incidencia de tornados. Su arquitectura está definida en la Figura 2.

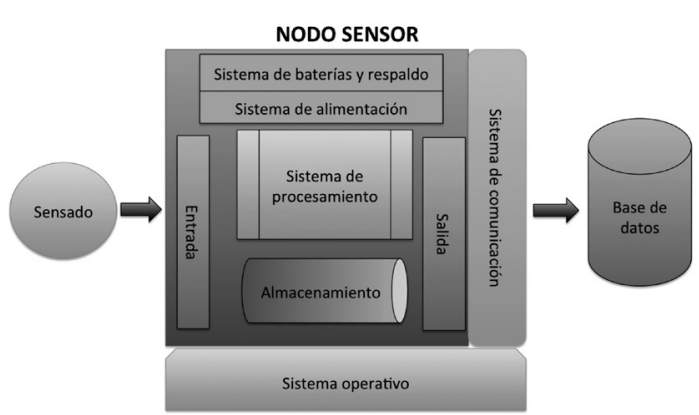

Figura 2. Diagrama de bloques del nodo sensor.

Para capturar estas señales se requiere de una plataforma de adquisición de datos que es el nodo sensor de la WSN tal como se muestra en la Figura 2. En ese sentido, se revisaron tecnologías como las placas Arduino, TelosB, MICAZ o Waspmote de Libelium [43] determinándose 
usar la placa Waspmote de Libelium como plataforma de adquisición de datos, por la fácil integración con los sensores, su lenguaje de programación bastante conocido y la amplia y disponible documentación.

\section{Placa de adquisición de datos Waspmote}

Es la tecnología Arduino potencializada en software y hardware libre para aplicaciones concretas de WSN [44], por lo que se tiene acceso a la documentación de sus partes; sus características principales se esbozan en la Tabla 2 y una descripción gráfica de la placa se observa en la Figura 3.

Tabla 2. Características de plataforma Waspmote [43, 45-46].

\begin{tabular}{|c|c|}
\hline Elemento & Características \\
\hline Microcontrolador & Atmega 1281 \\
\hline Alimentación & $3.3 \mathrm{~V}-4.7 \mathrm{~V}$ \\
\hline Entradas / Salidas & $\begin{array}{l}7 \text { análogas / } 8 \text { in - out } \\
\text { digitales }\end{array}$ \\
\hline SRAM & $8 \mathrm{kB}$ \\
\hline EEPROM & $4 \mathrm{kB}$ \\
\hline FLASH & $128 \mathrm{kB}$ \\
\hline Reloj & $32 \mathrm{kHz}$ \\
\hline $\begin{array}{l}\text { Rango de } \\
\text { temperatura de } \\
\text { operación }\end{array}$ & $-10^{\circ} \mathrm{C} \mathrm{a}+65^{\circ} \mathrm{C}$ \\
\hline $\begin{array}{l}\text { Consumo } \\
\text { energético }\end{array}$ & $\begin{array}{l}\text { Estado encendido: } 15 \mathrm{~mA} \\
\text { Estado bajo consumo } \\
\text { energético (Sleep): } 55 \mu \mathrm{A} \\
\text { Estado bajo consumo } \\
\text { energético profundo (Deep } \\
\text { Sleep): } 55 \mu \mathrm{A} \\
\text { Hibernación: } 0,07 \mu \mathrm{A}\end{array}$ \\
\hline $\begin{array}{l}\text { Sistema de } \\
\text { comunicación }\end{array}$ & $\begin{array}{l}\text { Integración de módulos Digi } \\
\text { Xbee S1/S2/PRO } \\
\text { Módulos GSM - 3G - GPS } \\
\text { - LoRa }\end{array}$ \\
\hline $\begin{array}{l}\text { Placas para } \\
\text { aplicaciones }\end{array}$ & $\begin{array}{l}\text { Placa para monitoreo de agua } \\
\text { Placa para ciudades } \\
\text { inteligentes } \\
\text { Placa para parqueo inteligente } \\
\text { Placa para agricultura } \\
\text { Placa para circuito de } \\
\text { corriente } \\
\text { Placa para sistemas de video } \\
\text { cámara } \\
\text { Placa para monitoreo } \\
\text { inteligente } \\
\text { Placa para prototipaje }\end{array}$ \\
\hline
\end{tabular}

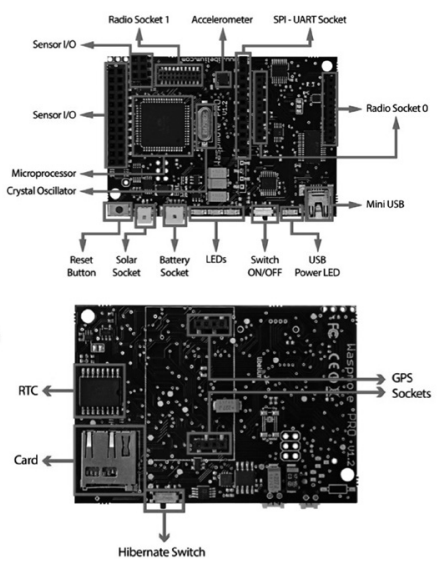

Figura 3. Vista superior y posterior con módulos de comunicación de la placa Libelium Waspmote [46].

Para realizar las mediciones de las variables ambientales escogidas se seleccionó la placa de agricultura que permite la integración de los sensores adecuados para esta función.

\section{Placa de agricultura}

Esta placa monitorea variables ambientales y se aplica a sistemas agrícolas en granjas [47] y a las observaciones meteorológicas [48] entre otras. Está diseñado para que se acoplen los sensores de temperatura, humedad relativa, presión atmosférica, luminosidad, velocidad y dirección del viento, cantidad de lluvia, sensor de humedad del suelo y radiación ultravioleta. En la Figura 4 se detallan los principales puertos de conexión de esta plataforma.

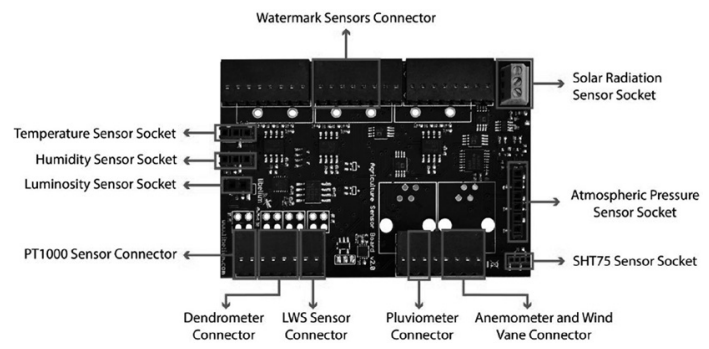

Figura 4. Puertos de conexión para los sensores de la placa de Agricultura Libelium [47-48].

\section{Sensores utilizados en la captación de variables atmosféricas}

Se detallan las principales características de los sensores de variables atmosféricas en la Tabla 3, 
Tabla 3. Principales características de los sensores utilizados [49-51].

\begin{tabular}{|l|l|l|l|}
\hline \multicolumn{1}{|c|}{ SENSOR } & \multicolumn{1}{c|}{ Temperatura } & \multicolumn{1}{c|}{ Humedad relativa } & Presión atmosférica \\
\hline Modelo & $\mathrm{MCP} 700 \mathrm{~A}$ (análogo) & $808 \mathrm{H} 5 \mathrm{~V} 5$ (capacitivo) & MPX4115A \\
\hline Rango de medición & $0{ }^{\circ} \mathrm{C}$ a $70{ }^{\circ} \mathrm{C}$ & $0-100 \% \mathrm{RH}$ & $15 \mathrm{KPA}-115 \mathrm{kPA}$ \\
\hline Exactitud & $+/-2^{\circ} \mathrm{C}$ & $\pm 4 \% \mathrm{RH}$ & $\pm 1,5 \mathrm{~V}$ \\
\hline Salida & $10 \mathrm{mV} /{ }^{\circ} \mathrm{C}$ & $0,8 \mathrm{~V}-3,9 \mathrm{~V}$ & $0,2 \mathrm{~V}-4,8 \mathrm{~V}$ DC \\
\hline Alimentación & $2,2 \mathrm{~V}-5 \mathrm{~V}$ DC & $5 \mathrm{~V}$ DC & $5,35 \mathrm{~V}$ DC (Máx.) \\
\hline Consumo energético & $6 \mathrm{uA}-12 \mathrm{uA}$ & $0,38 \mathrm{~mA}-0,5 \mathrm{~mA}$ & $7 \mathrm{~mA}-10 \mathrm{~mA}$ \\
\hline
\end{tabular}

relacionadas a los tornados que se producen en la ciudad de Barranquilla y sus zonas aledañas.

Para medir la cantidad de lluvia, dirección y velocidad del viento se usa el instrumento WS-3000 [46], el que posee un anemómetro, una veleta de viento y un pluviómetro. El anemómetro tiene un rango de operación de $0-140 \mathrm{~km} / \mathrm{h}$, con una sensibilidad de $2,4 \mathrm{~km} / \mathrm{h}$ por vuelta. El recipiente del pluviómetro tiene una capacidad de $0,28 \mathrm{~mm}$ y al llenarse mide la cantidad de agua que se está generando en una precipitación. Los tres sensores de la estación WS3000 (Figura 5) tienen asociados códigos y librerías del fabricante para la lectura de los datos.

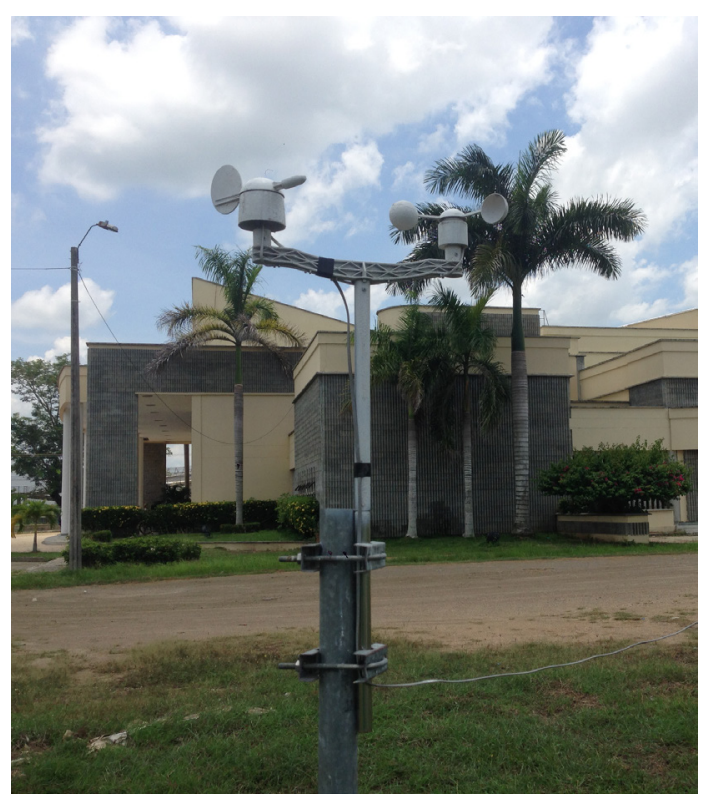

Figura 5. Estación meteorológica WS-3000.

\section{Interfaz de programación}

El Waspmote Pro IDE [46] es la herramienta de software que permite desarrollar códigos para aplicaciones específicas. Libelium tiene un lenguaje de programación basado en sketches para configurar los equipos Waspmote por medio de un editor de texto. El software Waspmote Pro IDE puede ser complementado con las librerías que se encuentran en el Waspmote API y soporta los lenguajes de programación $\mathrm{C}, \mathrm{C}++$, entre otros, para el desarrollo de las aplicaciones.

\section{Tecnología de comunicación}

Se usa el estándar ZigBee como protocolo de comunicación en la capa de red en las placas Waspmote, por lo que la capa de Zigbee opera sobre las únicas dos capas del estándar IEEE 802.15.4, la física y la de control de acceso al medio (MAC) $[52,53]$

Con los sensores se recolecta información de las condiciones meteorológicas locales previos a la generación de un tornado, para en un futuro generar una alerta a la población que pueda verse damnificada por este fenómeno.

\section{Descripción de las pruebas}

Por medio de la interfaz XCTU se llevó a efecto la programación del Waspmote y del módulo de comunicación. Inicialmente se realizaron pruebas de adquisición y transmisión de datos por puerto serial para verificar los valores adquiridos de los sensores. Posteriormente se realizó una prueba de envío de paquetes en una red punto a punto. Para esto se configuró el XBee receptor operado con el Gateway y cuya función es de Coordinador API. En la Figura 6 se muestra la configuración de los parámetros de coordinación de los XCTU.

El XBee transmisor ubicado en la tarjeta Waspmote, se configuró con la función de Router API usando el XCTU, y los parámetros de dirección de la red se ajustaron utilizando la herramienta de "Detección de enrutadores desconocidos por el coordinador" 


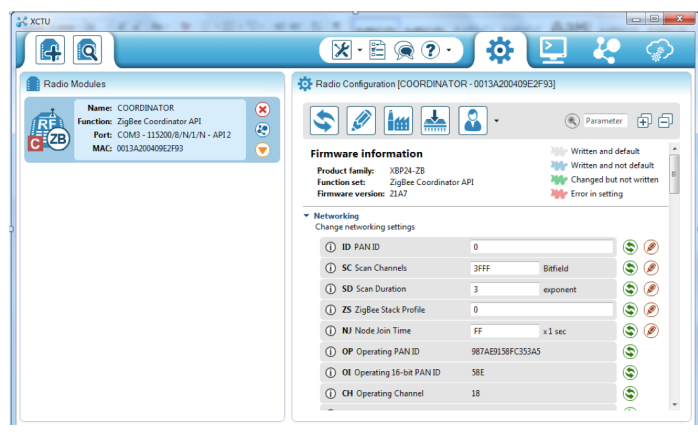

Figura 6. Configuración de los parámetros del coordinador en XCTU.

incluido en los ejemplos de Waspmote PRO IDE. En la Figura 7 se observa el reconocimiento de los módulos.

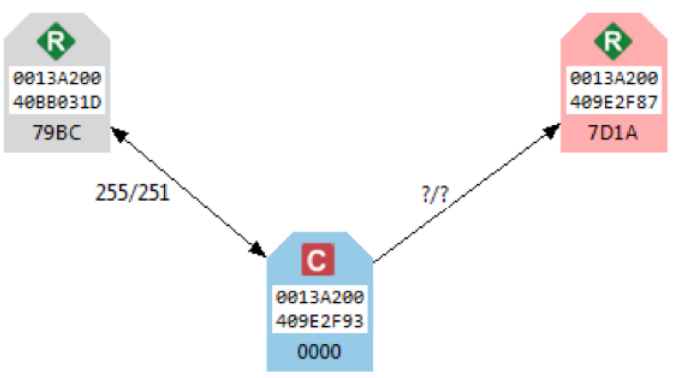

Figura 7. Diagrama de conexión punto a punto Visto en XCTU.

Después de mantener una conexión exitosa entre los dispositivos, se envió paquetes al nodo sumidero con los datos de los sensores de: temperatura, presión atmosférica, humedad relativa, anemómetro, veleta y pluviómetro.

En la etapa de prueba de cobertura del módulo de radio se ubica al primer nodo a $15 \mathrm{~m}$ de altura en un quinto piso, y a una distancia de 101,24 m con la Waspmote ubicada en el primer piso del mismo edificio. Se presentaron limitantes de conexión debido a obstáculos tales como los muros de las edificaciones. Se estableció la comunicación entre los sensores y un servidor que almacenó la información. Las pruebas fueron realizadas en las instalaciones de la Universidad de la Costa, CUC, en Barranquilla. En la Figura 8 se observan las componentes del despliegue en la prueba de campo dentro de las instalaciones de la Universidad de la Costa, con cada uno de los dispositivos que la integran.
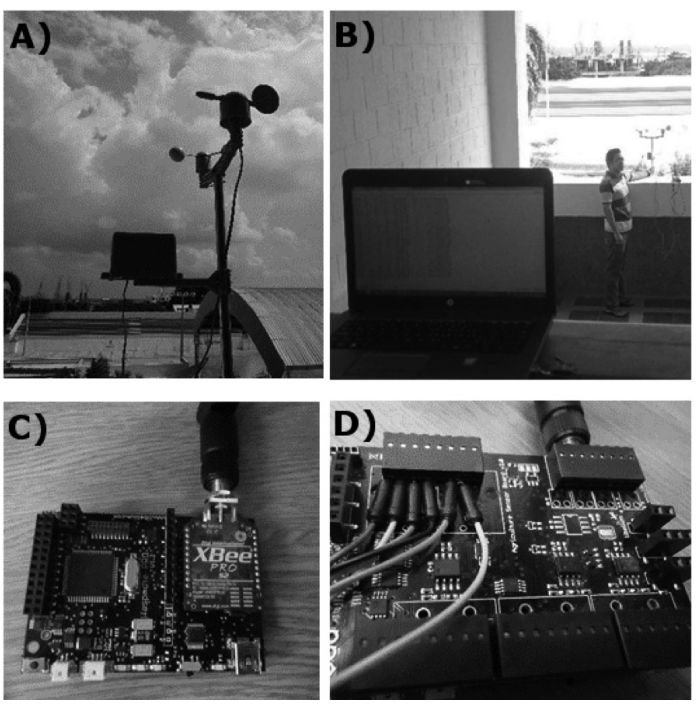

Figura 8. A) Estación meteorológica WS-3000; B) Comunicación entre la placa de agricultura de Libelium, el servidor y la estación meteorológica WS-3000; C) Módulo Zigbee; D) Módulo de comunicaciones Zigbee integrada con la placa de agricultura y la estación meteorológica WS-3000.

\section{RESULTADOS}

Luego del desarrollo de la metodología se ha elaborado un mapa con la ubicación de los puntos donde se han presentado con mayor número de frecuencia los tornados (uno por demarcación). Estos sirven de referencia para establecer la disposición geográfica que tendrán los nodos sensores para el despliegue y diseño del prototipo de sistema de monitoreo de condiciones meteorológicas locales.

A partir de la Figura 9 y la Tabla 1 se aprecia que en el departamento del Atlántico la mayor cantidad de tornados se presentaron en los municipios de Barranquilla y Soledad, por lo que la ubicación del prototipo del sistema de monitoreo se localiza en uno de estos municipios teniendo como centro base a la Universidad de la Costa con el nodo sumidero. 


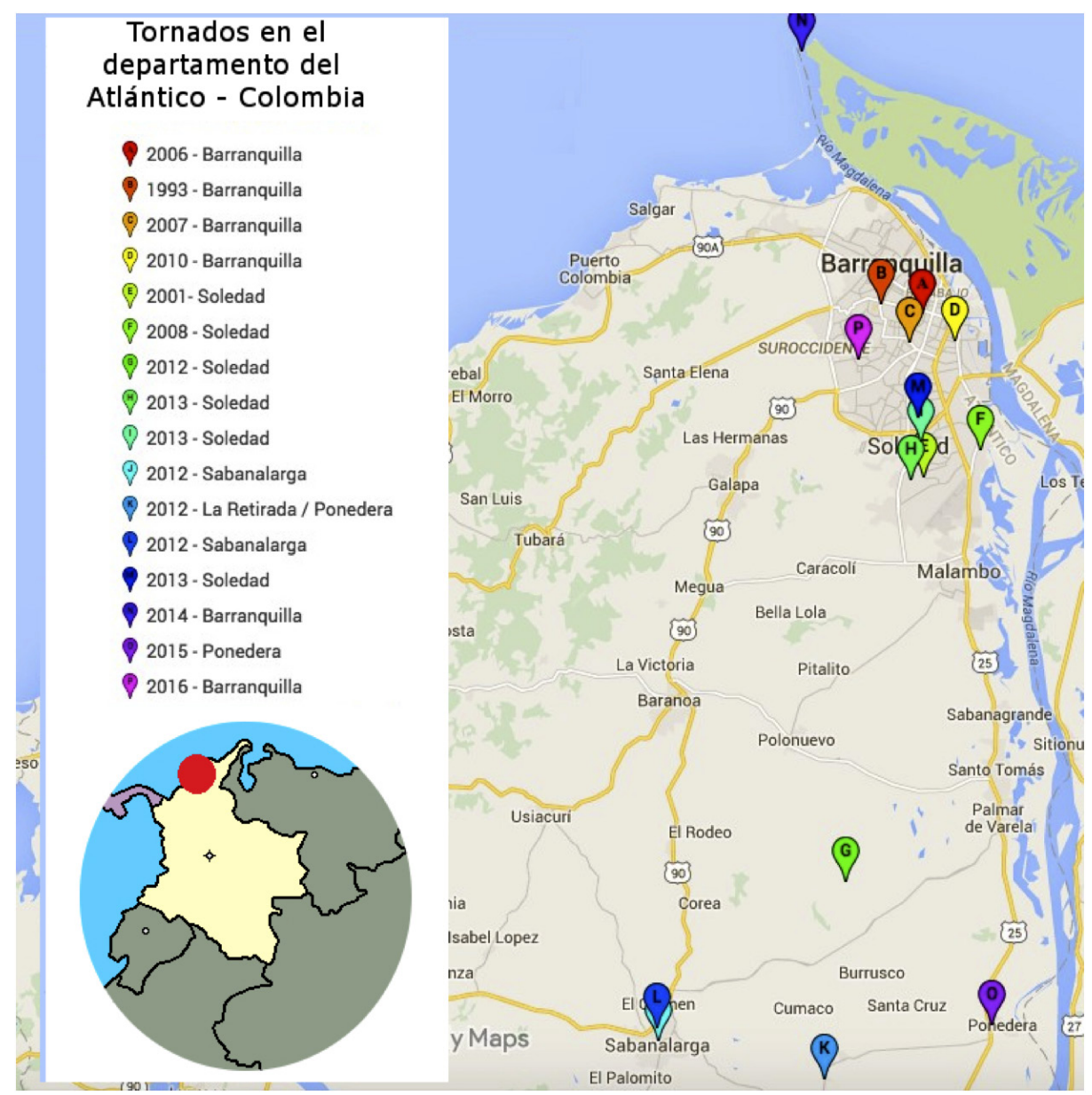

Figura 9. Mapa con ubicación de tornados suscitados en el departamento del Atlántico en los últimos años.

Un diagrama del diseño de la red se muestra en la Figura 10.

La Figura 10 muestra al prototipo operando entre la zona noroccidental y suroccidental del municipio de Barranquilla. Se simuló los enlaces inalámbricos usando el software Radio Mobile [54] que utiliza mapas de elevación digital que están disponibles de forma gratuita y el modelo de propagación Longley-Rice elaborado en el ITS (Instituto de Estados Unidos para la Ciencia Telecomunicaciones). Este modelo predice las transmisiones de radios troposférica y la pérdida sobre terrenos irregulares para un enlace de radio (de transmisor al receptor), y se conoce como el Modelo de Terreno Irregular (ITM). El modelo fue diseñado para frecuencias entre $20 \mathrm{MHz}$ y 20 $\mathrm{GHz}$ y longitudes de trayecto entre $1 \mathrm{~km}$ y 2.000

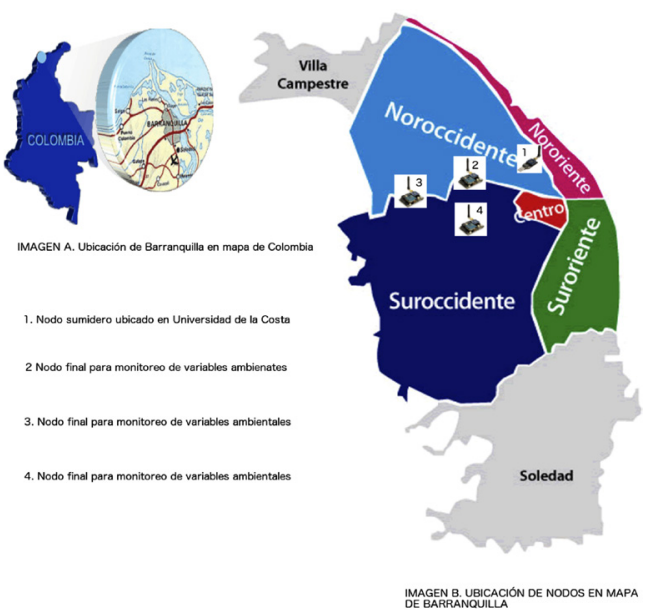

Figura 10. Ubicación de nodos del prototipo WSN para el monitoreo de las condiciones meteorológicas locales. 
km [55]. La topología simulada con Radio Mobile se muestra en la Figura 11.

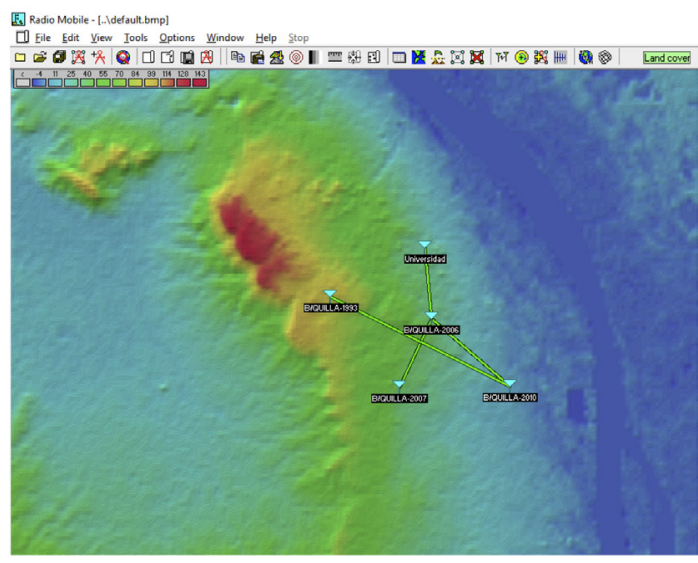

Figura 11. Topología simulada con el software Radio Mobile.

En la Figura 11 se modelan las estaciones meteorológicas interconectadas por una WSN de cinco puntos. Cuatro de ellas ubicadas en sectores donde se presentaron en años distintos presencia de tornados (1993, 2006, 2007 y 2010). El quinto punto corresponde al que se ubica en la Universidad de la Costa que recolecta y administra la información de la red.

Para esta simulación se considera que todos los nodos de la red usarán una antena omnidireccional de $5 \mathrm{dBi}$ de ganancia y el mismo transceptor, el XBee-PRO, cuyas características técnicas son apropiadas para enlaces kilométricos con línea de vista. Su sensibilidad de recepción es de $-100 \mathrm{dBm}$, y potencia de transmisión llega a los $18 \mathrm{dBm}$; trabaja en la banda libre ICM de $2.4 \mathrm{GHz}$, la que es escogida en la configuración de Radio Mobile, como se muestra en la Figura 12.

Los cuatro enlaces de la red propuesta corresponden a los puntos Barranquilla 1993 - Barranquilla 2010 (enlace 1); Barranquilla 2006 - Barranquilla 2007 (enlace 2); Barranquilla 2006 - Barranquilla 2010 (enlace 3); Barranquilla 2006 - Universidad de la Costa (enlace 4). Los resultados de las simulaciones para los cuatro enlaces se muestran en las Figuras 13, 14, 15 y 16.

En las Figuras 13, 14, 15, 16 el software Radio Mobile revela la factibilidad de los radioenlaces debido a que el obstáculo más cercano a cada enlace

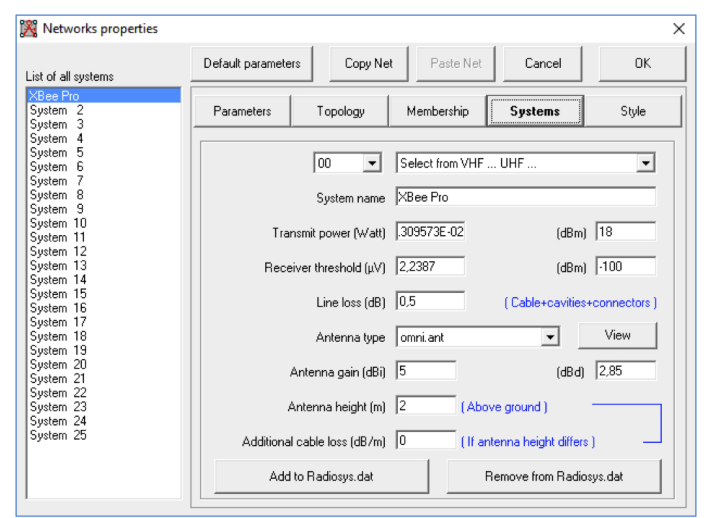

Figura 12. Configuración del módulo de radio en Radio Mobile.

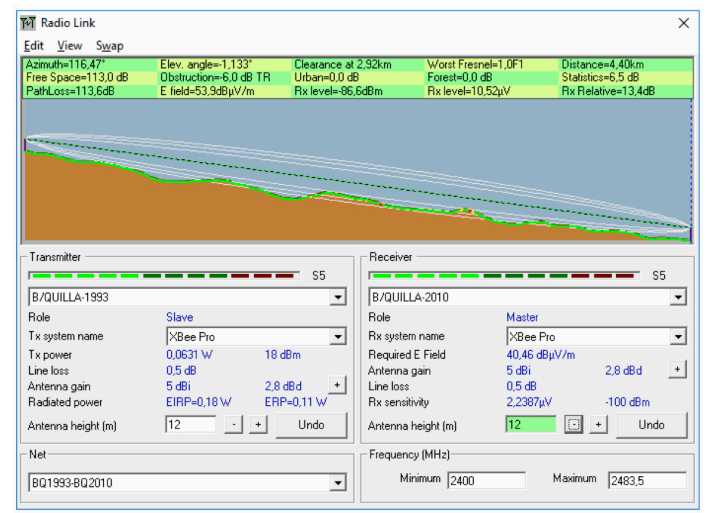

Figura 13. Simulación en el entorno Radio Mobile para el enlace 1.

inalámbrico está a una distancia igual al valor del radio de la primera zona de Fresnel (Worst Fresnel) y el margen de la recepción relativa es superior a los $10 \mathrm{~dB}$ [56] que indica la estabilidad de los enlaces.

Para las mediciones de las variables se tomaron 116 muestras en uno de los nodos con intervalos de 30s para analizar la consistencia de los datos durante el monitoreo de los sensores instalados. Las mediciones obtenidas por los sensores fueron estables, y los resultados obtenidos son mostrados en las Figuras 17, 18, 19 y 20.

\section{CONCLUSIONES Y TRABAJO FUTURO}

El uso de la red de sensores inalámbricos (WSN) para monitorear las variables relacionadas con las condiciones meteorológicas locales en zonas donde se 


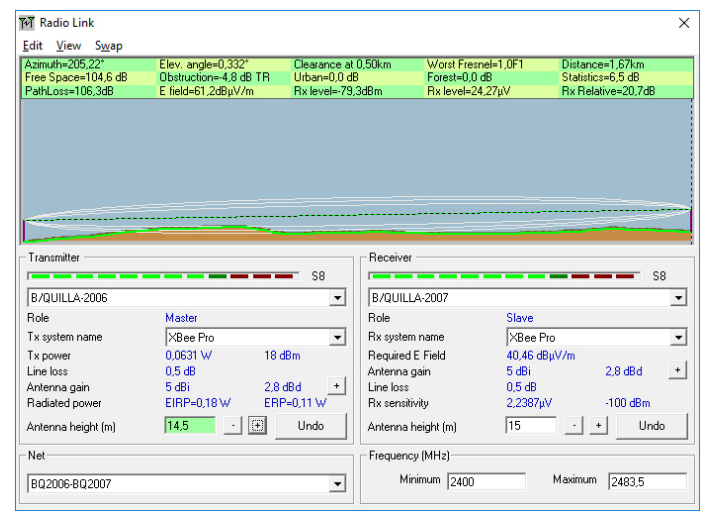

Figura 14. Simulación en el entorno Radio Mobile para el enlace 2.

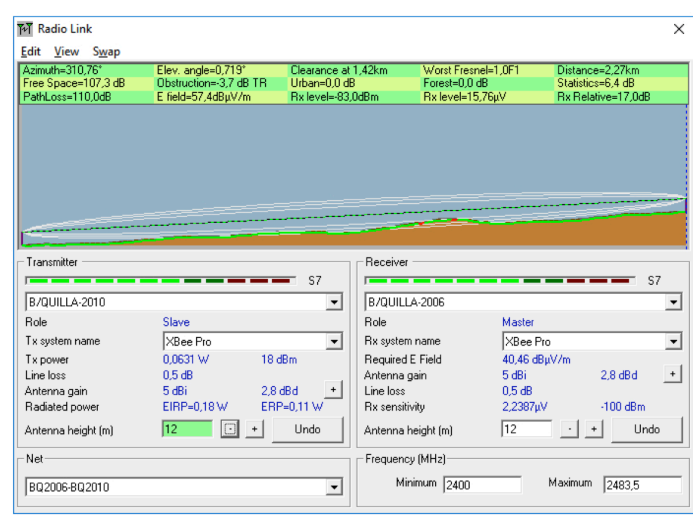

Figura 15. Simulación en el entorno Radio Mobile para el enlace 3 .

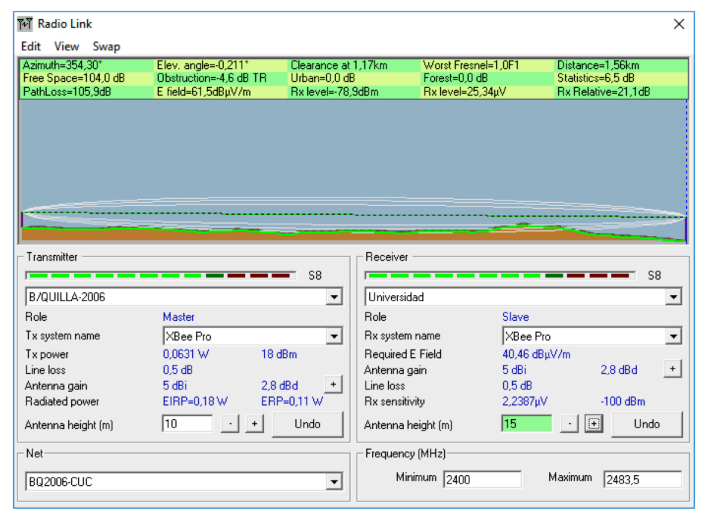

Figura 16. Simulación en el entorno Radio Mobile para el enlace 4.

ha dado la aparición de los tornados que se producen en la ciudad de Barranquilla es viable desde el punto técnico, y su despliegue servirá como una solución

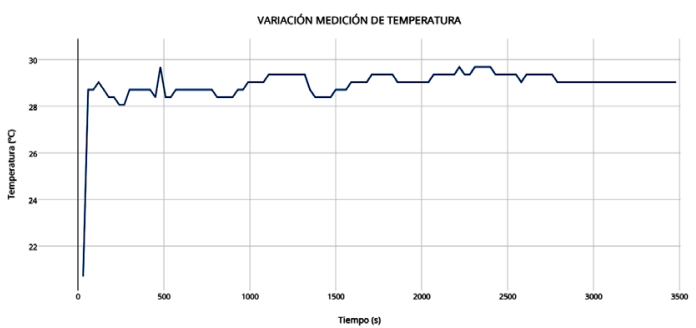

Figura 17. Toma de datos para el sensor de temperatura.

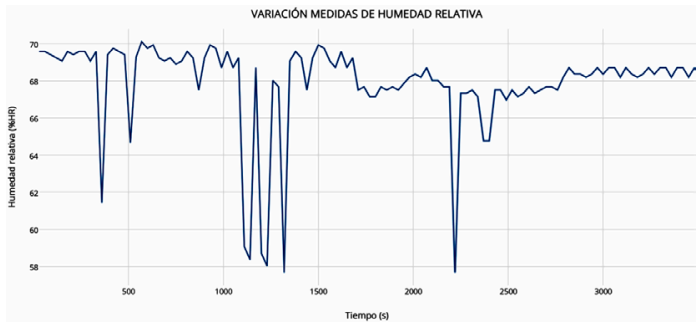

Figura 18. Toma de datos para el sensor de humedad relativa.

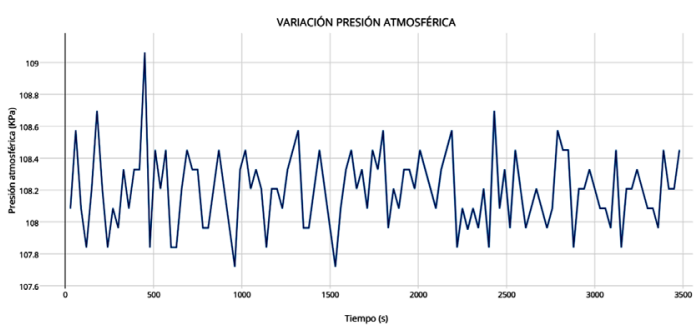

Figura 19. Toma de datos para el sensor de presión atmosférica.

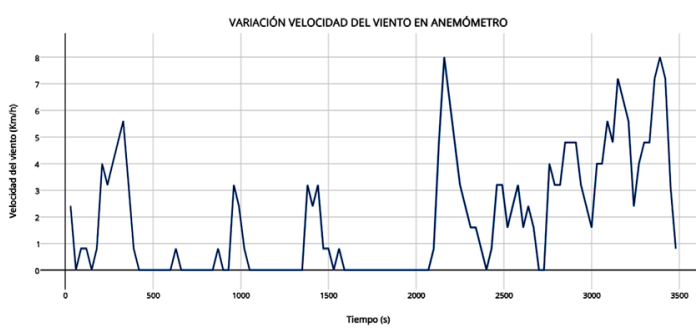

Figura 20. Toma de datos para el anemómetro.

para el estudio de las condiciones meteorológicas locales y la prevención de los accidentes en las personas por este tipo de fenómenos naturales. Por otro lado, la creación de una base de datos que albergue información del número de tornados que se forman a lo largo del año servirá para entenderlos 
mejor ya que muchas veces no son registrados sino solo hasta cuando estos provocan estragos evidentes en las zonas de la ciudad por donde atraviesan. El siguiente paso, luego del despliegue permanente del sistema de sensores y con la disposición de los datos acumulados durante largos períodos de meses o años, será la creación de algoritmos predictivos que puedan con un alto porcentaje de probabilidad determinar o estimar la aparición de algún tornado.

\section{AGRADECIMIENTOS}

Los autores agradecemos a la Asociación Universitaria Iberoamericana de Postgrado (AUIP) por su apoyo en el trabajo de investigación. Además se extienden los agradecimientos al Departamento Administrativo de Ciencia, Tecnología e Innovación (COLCIENCIAS) a través de su apoyo por medio de su convocatoria Joven Investigador.

\section{REFERENCIAS}

[1] Z. F. Yang, P. Sarkar, and H. Hu. "An experimental study of a high-rise building model in tornado-like winds". Journal of Fluids and Structures Volumen 27. Número 4, pp. 471-486. Mayo de 2011. ISSN: 08899746. DOI: http://dx.doi.org/10.1016/j. jfluidstructs.2011.02.011.

[2] A.A. El Damatty and A. Hamada. "F2 tornado velocity profiles critical for transmission line structures". Engineering Structures. Volumen 106, pp. 436-449. Enero 2016. ISSN: 0141-0296. DOI: http:// dx.doi.org/10.1016/j.engstruct.2015.10.020.

[3] P.T. Nastos and J.T. Matsangouras, "Tornado activity in Greece within the 20th century". Advances in Geosciences. Volumen 26, pp. 49-51. Julio 2010. ISSN: 16807359. DOI:10.5194/adgeo-26-49-2010.

[4] A. Lampis. "Vulnerabilidad y adaptación al cambio climático: debates acerca del concepto de vulnerabilidad y su medición”. Revista Colombiana de Geografía. Volumen 22. Número 2, pp. 17-33. 2013. ISSN: 22565442. DOI: $10.15446 / \mathrm{rcdg}$.

[5] S.A. Yershin and A.K. Yershina. "Tornado, twisters - secondary currents within the atmosphere". Mathematics and Computers in Simulation. Volumen 67, pp. 327-334. Diciembre de 2004. ISSN: 0378-4754. DOI: http://dx.doi.org/10.1016/j. matcom.2004.06.008

[6] S.S. Banik, H.P. Hong, and GA Kopp. "Tornado hazard assessment for southern Ontario". Canadian Journal of Civil Engineering. Volumen 7, pp. 830-842. 2007. ISSN: 0315-1468. DOI: http://dx.doi. org/10.1139/107-001.

[7] A.M. Goliger and R.V. Milford. "A review of worldwide occurrence of tornadoes". Journal of Wind Engineering and Industrial Aerodynamics. Volumen 74-76, pp. 111-121. Abril de 1998. ISSN: 0167-6105. DOI: http:// dx.doi.org/10.1016/S0167-6105(98)00009-9.

[8] D. Samanthi, S.C. Wirasinghe, and J. Ruwanpura. "Comparison of the Canadian and US tornado detection and warning systems". Natural Hazards. Volumen 66. Número 1, pp. 117-137. Marzo de 2013. ISSN: 15730840. DOI: 10.1007/s11069-012-0168-7.

[9] Dane. "Demografía y población Proyecciones de población". Agosto de 2015. Consultado 30 de junio del 2016. Disponible en : http://www.dane.gov. co/index.php/poblacion-y-demografia/ proyecciones-de-poblacion.

[10] K. Villadiego and M.A. Velay-Dabat. "Outdoor thermal comfort in a hot and humid climate of Colombia: A field study in Barranquilla". Building and Environment. Volumen 75, pp. 142-152. Mayo de 2014. ISSN: 03601323. DOI: http://dx.doi.org/10.1016/j. buildenv.2014.01.017.

[11] L. Lemon and C. Doswell III. "Severe Thunderstorm Evolution and Mesocyclone Structure as Related to Tornadogenesis". Monthly Weather Review. Volumen 107. Número 9, pp. 1184-1197. Septiembre de 1979. DOI: dx.doi. org/10.1175/1520-0493(1979)107<1184:STE AMS >2.0.CO;2.

[12] H. Brooks and C. Doswell III. "Some aspects of the international climatology of tornadoes by damage classification". Atmospheric Research.Volumen 56. Número 1-4, pp. 191-201. Enero de 2001. ISSN: 01698095. DOI: http://dx.doi.org/10.1016/ S0169-8095(00)00098-3.

[13] B. Feuerstein, N. Dotzek, and J. Grieser "Assessing a Tornado Climatology from 
Global Tornado Intensity Distributions". Journal of Climate. Volumen 18. Número 4, pp. 585-596. Febrero de 2005. DOI: http:// dx.doi.org/10.1175/JCLI-3285.1.

[14] G. Piñeres-Espitia "Tecnologías para el estudio y prevención de tornados en el contexto del departamento del Atlántico". Scientia et Technica. Volumen 2. Número 51, pp. 166174. Agosto de 2012. ISSN: 0122-1701.

[15] F. Montoya, J. Gómez, A. Cama-Pinto, A. Zapata-Sierra, F. Martínez, J. De La Cruz, F. Manzano-Agugliaro. "A monitoring system for intensive agriculture based on mesh networks and the android system". Computers and Electronics in Agriculture. Volumen 99, pp. 14-20. Noviembre de 2013. ISSN: 0168-1699. DOI: http://dx.doi. org/10.1016/j.compag.2013.08.028.

[16] A. Cama-Pinto, G. Pineres-Espitia, R. Zamora-Musa, M. Acosta-Coll, J. Caicedo-Ortiz, J. Sepúlveda-Ojeda. "Design of a wireless sensor network for monitoring of flash floods in the city of Barranquilla Colombia”. Ingeniare: Revista Chilena De Ingeniería. Volumen 24 (4), pp. 581-599. Octubre 2016. ISSN: 0718-3305. https://dx.doi.org/10.4067/ S0718-33052016000400005.

[17] G. Salini-Calderón. "Estudio acerca del material particulado emitido en ciudades de tamaño medio al sur de Santiago de Chile". Revista Inge CUC. Volumen 10. Número 1, pp. 97-108. Junio de 2014. ISSN 2382-4700.

[18] M. Lazarescu. "Design of a WSN Platform for Long-Term Environmental Monitoring for IoT Applications". IEEE Journal on Emerging and Selected Topics in Circuits and Systems. Volumen 3. Número 1, pp. 45-54. Marzo de 2013. ISSN: 2156-3357. DOI: 10.1109/JETCAS.2013.2243032.

[19] G. Piñeres-Espitia. "Acercamiento tecnológico a la aparición de tornados en Barranquilla". Educosta, pp. 31-112. Barranquilla, Colombia. ISBN: 978-9588710-45-7. 2010.

[20] A. Artuz. "El tiempo: Tornados son posibles en Barranquilla según estudio". Octubre del 2012. Consultado el 11 de julio del 2016. Disponible en: http://www.eltiempo.com/ archivo/documento/CMS-12288044.
[21] R. M. Wakimoto and W. James W., "Nonsupercell Tornadoes," AMS J. Online, pp. 1113-1140, 2013

[22] M. Obredor. "El Heraldo: lo que vivimos fue como volver a nacer". Septiembre de 2012. Consultado julio 06 del 2016. Disponible en: http://www.elheraldo.co/galeria-fotos/81434/ local/lo-que-vivimos-fue-como-volver-anacer-81434.

[23] Alcaldía de Sabanalarga. "Tornado deja un muerto y 107 heridos en Sabanalarga". 0 de junio del 2012. Consultado julio 6 del 2016. Disponible en: http://www. sabanalarga-atlantico.gov.co/noticias. shtml?apc $=$ Cnxx-1-\&x=2763455.

[24] Noticosta. "Alcaldía de Soledad Finalizo Entrega de Materiales a Damnificados". Septiembre de 2013. Consultado 7 de julio del 2016. Disponible en: http:// www.noticosta.com/NewsFull/vernoticia. php?idNoticia $=5565 \&$ category $=22$

[25] El Heraldo. "Tromba marina fue vista este miércoles en el norte de Barranquilla”. Noviembre de 2014. Consultado 6 de julio del 2016. Disponible en: http://www.elheraldo.co/local/ tromba-marina-fue-vista-este-miercolesen-el-norte-de-barranquilla-173801.

[26] C. Polo. "El Heraldo: 92 casas fueron afectadas por vendaval en Atlántico". Septiembre de 2015. Consultado 6 de julio del 2016. Disponible en: http://www.elheraldo. co/local/92-casas-fueron-afectadas-porvendaval-en-atlantico-215230.

[27] M. Sáez. "El Heraldo: Vendaval en el Suroccidente deja 36 casas destechadas". Abril del 2016. Consultado 6 de julio del 2016. Disponible en: http://www.elheraldo. co/local/vendaval-en-el-suroccidente-deja36-casas-destechadas-256636.

[28] F. Tatom, K. Knupp, and S. Vitton. "Tornado Detection Based on Seismic Signa”. Journal of Applied Meteorology and Climatology. Volumen 34. Número 2, pp. 572-582. Febrero de 1995. DOI: http://dx.doi. org/10.1175/1520-0450(1995)034<0572:TD BOSS>2.0.CO;2 Número.

[29] P. Simeonov, L. Bocheva, and I. Gospodinov. "On space-time distribution of tornado events in Bulgaria (1956-2010) with brief analyses of 
two cases" Atmospheric Research. Volumen 123, pp. 61-70. Abril de 2013. ISSN 01698095. DOI: http://dx.doi.org/10.1016/j. atmosres.2012.07.003.

[30] M. Avvenuti, S. Cresci, A. Marchetti, and M. Tesconi. "Earthquake emergency management by social sensing". IEEE International Conference on Pervasive Computing and Communications Workshops (PERCOM Workshops), pp. 587-592. Budapest, Hungría. Marzo de 2014.

[31] P. Chan, J. Wurman, C. Shun, P. Robinson, and K. Kosiba. "Application of a method for the automatic detection and GroundBased Velocity Track Display (GBVTD) analysis of a tornado crossing the Hong Kong International Airport". Atmospheric Research. Volumen 106, pp. 18-29. Marzo del 2012. ISSN: 0169-8095. DOI: http:// dx.doi.org/10.1016/j.atmosres.2011.10.010.

[32] E. Rasmussen, J. Straka, R. Davies-Jones, C. Doswell III, F. Carr, M. Eilts, and D.R. MacGorman. "Verification of the Origins of Rotation in Tornadoes Experiment: VORTEX" Bulletin of the American Meteorologycal Society. Volumen 75. Número 6, pp. 9951006. Junio de 1994. DOI: http://dx.doi. org/10.1175/1520-0477(1994)075<0995:VO TOOR $>2.0 . \mathrm{CO} ; 2$.

[33] S. Verbout, H. Brooks, L. Leslie, and D. Schultz. "Evolution of the U.S. Tornado Database: 1954-2003". Weather and Forecasting. Volumen 21. Número 1, pp. 86-93. Febrero de 2006. DOI: http:// dx.doi.org/10.1175/WAF910.1.

[34] P. Tari, R. Gurka, and H. Hangan. "Experimental investigation of tornado-like vortex dynamics with swirl ratio: The mean and turbulent flow fields". Journal of Wind Engineering and Industrial Aerodynamics. Volumen 98. Número 12, pp. 936-944. Noviembre de 2010. DOI: 10.1016/j. jweia.2010.10.001.

[35] A. Dasgupta and D. Callahan. "Vehicular tornado warnings". IEEE 55th Vehicular Technology Conference. Volumen 2, pp. 590-592. Mayo del 2002. doi: 10.1109/ VTC.2002.1002551.

[36] P. Simeonov and C. G. Georgiev. "Severe wind/hail storms over Bulgaria in 1999-2001 period: synoptic- and meso-scale factors for generation". Atmospheric Research. Volumen 67-68, pp. 629-643. Julio-Septiembre de 2003. ISSN: 0169-8095. DOI: http://dx.doi. org/10.1016/S0169-8095(03)00077-2.

[37] C.A. Aguirre, J. Martínez, F. Martínez, A. Brizuela, and M. Moyano. "Simulación de las grandes escalas de flujo turbulento sobre una colina de suave pendiente". Serie Mecánica Computacional, Argentina. Volumen 16, pp. 1215-1235. 2007. ISSN 1666-6070.

[38] R. Borde, M. Doutriaux-Boucher, G. Dew, and M. Carranza. "A Direct Link between Feature Tracking and Height Assignment of Operational EUMETSAT Atmospheric Motion Vectors", Journal of Atmospheric and Oceanic Technology. Volumen 31. Número 1, pp. 33-46. Enero de 2014. DOI: http://dx.doi. org/10.1175/JTECH-D-13-00126.1.

[39] L. Hongmei and H. Hengyang. "Wireless Sensor Network Deployment Using an Optimized Artificial Fish Swarm Algorithm". 2012 International Conference on Computer Science and Electronics Engineering (ICCSEE), pp. 90-94. Hangzhou, China. 2012. doi: 10.1109/ICCSEE.2012.453.

[40] R. Klauck and M. Kirsche. "Combining mobile XMPP entities and cloud services for collaborative post-disaster management in hybrid network environments". Mobile Networks and Applications. Volumen 18. Número 2, pp. 253-270. Agosto de 2012. ISSN 1572-8153. DOI: 10.1007/s11036-012-0391-1.

[41] S. Manfredi. "Design of a multi-hop dynamic consensus algorithm over wireless sensor networks". Control Engineering Practice. Volumen 21. Número 4, pp. 381-394. Abril de 2013. ISSN: 09670661. DOI: http://dx.doi.org/10.1016/j. conengprac.2012.12.001.

[42] G. Lavold. "Developing and Using the Work Breakdown Structure". John Wiley \& Sons, Inc. Segunda edicion, pp. 303 -323. Hoboken, New Jersey, USA. ISBN: 9780471293842. 1997.

[43] C. Pham, V. Lecuire, and J.-M. Moureaux. "Performances of multi-hops image transmissions on IEEE 802.15.4 Wireless Sensor Networks for surveillance 
applications". 2013 IEEE 9th International Conference on Wireless and Mobile Computing, Networking and Communications (WiMob), pp. 477-484. Lyon, Francia. 2013. doi: 10.1109/WiMOB.2013.6673402.

[44] Digi, "Digi connect with confidence". Agosto de 2015. Consultado junio 4 del 2016. Disponible en: http://www.digi.com/.

[45] S. Janos and I. Matijevics. "Simulation and implementation of mobile measuring robot navigation algorithms in controlled microclimatic environment using WSN". 2011 IEEE 9th International Symposium on Intelligent Systems and Informatics, pp. 275-279. Subotica, Serbia. 2011. doi: 10.1109/SISY.2011.6034337.

[46] Libelium. "Libelium". Agosto de 2015. Consultado junio 8 del 2016. Disponible en: http://www.libelium.com/.

[47] A. Kaloxylos, R. Eigenmann, F. Teye, Z. Politopoulou, S. Wolfert, C. Shrank, M. Dillinger, I. Lampropoulou, E. Antoniou, L. Pesonen, H. Nicole, F. Thomas, N. Alonistioti, and G. Kormentzas. "Farm management systems and the Future Internet era" Computers and Electronics in Agriculture. Volumen 89, pp. 130-144. Noviembre de 2012. ISSN: 0168-1699. DOI: http://dx.doi. org/10.1016/j.compag.2012.09.002..

[48] P. Thornton, S. Running, and M. White. "Generating surfaces of daily meteorological variables over large regions of complex terrain". Journal of Hydrology. Volumen 190. Número 3-4, pp. 214-251. Marzo de 1997. ISSN: 0022-1694. DOI: http://dx.doi. org/10.1016/S0022-1694(96)03128-9.

[49] Sparkfun. "Sparkfun". Agosto de 2015. Consultado 12 de junio del 2016. Disponible en: https://www.sparkfun.com/.

[50] E. TEM. "TEM Electronics components". Septiembre de 2015. Consultado 13 de junio del 2016. Disponible en: http://www.tme.eu/ en/details/sens-808h5v5/humidity-sensors/.

[51] Freescale, "Freescale". Agosto de 2015. Consultado 14 de junio del 2016. Disponible en: http://ir.freescale.com/investor-relations/ press-release-archive/2009.aspx.

[52] L. Ruiz-García, P. Barreiro, and J.I. Robla. "Performance of ZigBee-Based wireless sensor nodes for real-time monitoring of fruit logistics". Journal of Food Engineering. Volumen 87, pp. 405-415. Agosto de 2008. ISSN: 0260-8774. DOI: http://dx.doi. org/10.1016/j.jfoodeng.2007.12.033.

[53] G. Piñeres-Espitia and Á. Mejía. "Plataformas tecnológicas aplicadas al monitoreo climático". Revista Prospectiva. Volumen 11. Numero 2, pp. 78-87. Diciembre de 2013. ISSN. 2216-1368. DOI: http://dx.doi. org/10.15665/rp.v11i2.42

[54] O. Fratu, A. Martian, R. Craciunescu, A. Vulpe, S. Halunga, Z. Zaharis, P. Lazaridis, S. Kasampalis. "Comparative study of Radio Mobile and ICS Telecom propagation prediction models for DVB-T". IEEE International Symposium on Broadband Multimedia Systems and Broadcasting, BMSB, pp. 1-6. Agosto del 2015. DOI: 10.1109/BMSB.2015.7177260

[55] J.S. Tietjen. "Anita longley's legacy: The longley-rice model-still going strong after almost 50 years". IEEE Antennas and Propagation Magazine. Volumen 55. № 3, pp. 237-240, June 2013. doi: 10.1109/ MAP.2013.6586676

[56] M. Zennaro, A. Bagula, D. Gascon. "Noveleta, A.B. Long distance wireless sensor networks: Simulation vs reality". Proceedings of the 4th ACM Workshop on Networked Systems for Developing Regions, NSDR ' 10 , art. $\mathrm{N}^{\circ} 12$. DOI: $10.1145 / 1836001.1836013$ 